\title{
O fator de crescimento de fibroblasto básico melhora a cicatrização de anastomoses duodenais em $\operatorname{ratos}^{1}$
}

\author{
Aldo da Cunha Medeiros ${ }^{2}$ \\ Antônio Medeiros Dantas Filho ${ }^{3}$ \\ Tertuliano Aires Neto ${ }^{3}$ \\ Francisco Pignataro Lima ${ }^{4}$ \\ Ítalo Medeiros de Azevêdo ${ }^{5}$ \\ Silvana Gomes Alves ${ }^{6}$
}

\begin{abstract}
Medeiros AC, Dantas Filho AM, Aires Neto T, Lima FP, Azevedo IM, Alves SG. O fator de crescimento de fibroblasto básico melhora a cicatrização de anastomoses duodenais em ratos. Acta Cir Bras [online] 2003 vol 18 suppl 1. Disponível em www.scielo.br/acb.
\end{abstract}

RESUMO: Objetivo: Avaliar as alterações histológicas, e o ganho de resistência em anastomoses duodenais tratadas com fator de crescimento de fibroblasto básico (FGFb). Métodos: Vinte ratos da raça Wistar foram submetidos a secção transversal do duodeno, seguida de anastomose. Os animais foram divididos em 4 grupos de 5 animais cada: A1 e A2 (experimentais), nos quais foi aplicado FCFb sobre a anastomose logo após seu término; e B1 e B2 (controles), nos quais foi administrada solução salina sobre a zona de anastomose. Os roedores foram mortos com superdose de anestésico, sendo $\mathrm{A} 1, \mathrm{~B} 1$ no $5^{\circ}$ dia e $\mathrm{A} 2, \mathrm{~B} 2$ no $7^{\circ}$ dia de pós-operatório. Foi feita avaliação quanto à resistência das anastomoses à pressão e análise da densidade média dos achados histopatológicos com auxílio do sistema digitalizado Image proPlus. Resultados: No grupo Al a pressão suportada pelas anastomoses foi de $52 \pm 14,4 \mathrm{mmHg}$ e no grupo A2 $140 \pm 34,8 \mathrm{mmHg}$. Em $\mathrm{B} 1$ a pressão atingiu $33,6 \pm 15,2 \mathrm{mmHg}$ e as anastomoses do grupo B2 suportaram pressão $105 \pm 30,3$. No grupo Al a densidade média dos elementos histopatológicos foi de $93 \pm 9,3$ e A2 atingiu 181,8 $\pm 27,6$. Nos grupos de controle B1 e B2 as densidades médias foram 67,6 $\pm 16,7$ e $101 \pm 12,9$ respectivamente. A análise estatística revelou diferença significante entre nos dados dos grupos experimentais e controles $(\mathrm{p}<0,05)$. Conclusão: a aplicação tópica do $\mathrm{FCFb}$ foi capaz de aumentar a resistência das feridas do duodeno suturadas e observadas após 5 e 7 dias de evolução. Estimulou a neovascularização, a formação de fibroblastos e de fibras colágenas, melhorando os escores histológicos em relação ao controle.

DESCRITORES: Fator de crescimento de fibroblasto. Cicatrização. Anastomose. Duodeno. Resistência. Colágeno.

\section{INTRODUÇÃO}

Os fatores de crescimento têm múltiplas funções, detacando-se a estimulação da divisão celular, angio- gênese, quimiotaxia, indução ou inibição da diferenciação celular, transformação ou indução da síntese de proteínas $^{1,2,3,4}$. Estas substâncias polipeptídicas têm grande variedade de fontes e alvos celulares. Dentre os

1. Trabalho do Núcleo de Cirurgia Experimental da UFRN (Apoiado pelo CNPq).

2. Prof. Dr. do Departamento de cirurgia da UFRN; Chefe do Núcleo de Cirurgia Experimental; Pesquisador nível I do CNPq

3. Prof. do Departamento de Cirurgia-UFRN; aluno do Programa de Pós-graduação.

4. Prof. Mestre do Departamento de Patologia-UFRN.

5. Aluno do Departamento de Estatística-UFRN.

6. Aluna bolsista de Iniciação Científica, $\mathrm{CNPq}-\mathrm{PIBIC}$. 
fatores de crescimento, os que parecem ter mais destaque na cicatrização das feridas são os fatores de crescimento de fibroblasto (FCF), sendo que o FCF de reação básica $(\mathrm{FCFb})$ exerce um maior número de reações fisiológicas ${ }^{5}$. As principais fontes destas citocinas são macrófagos, linfócitos e plaquetas ${ }^{6,7}$. Para Davidson ${ }^{8}$ os fatores de crescimento são essencialmente angiogênicos, promovendo portanto o crescimento vascular, aspecto extremamente desejável em um processo cicatricial. Além disso, eles exercem ação indireta na síntese de colágeno, proteína fibrosa que dá resistência às anastomoses. $\mathrm{Os} \mathrm{FCFb}$ tanto podem estimular como inibir os fibroblastos, tornando-se importante mecanismo de retroalimentação na síntese e destruição do colágeno, assim como da própria cicatrização das feridas $^{9,10}$.

Tomando por base as características anatômicas e fisiológicas do duodeno como um órgão sujeito a incidência não desprezível de fístulas e deiscências pósoperatórias, e os efeitos do $\mathrm{FCFb}$, foi realizado estudo experimental em ratos com o propósito de testar a ação desta citocina na cicatrização de anastomoses do duodeno, consideradas como anastomoses de risco. $\mathrm{O}$ trabalho tem o objetivo de estudar as alterações histológicas causadas pelos FGFb nas anastomoses duodenais e observar o seu ganho de resistência sob a ação dos $\mathrm{FGFb}$.

\section{MÉTODOS}

Reagentes: Foi utilizado o Fator de Crescimento de Fibroblastos básico $(\mathrm{FCFb})$ associado ao colágeno solúvel, como veículo, com a finalidade de facilitar a aderência do mesmo ao duodeno em estudo (Reagentes de procedência SigmaÒ).

Procedimentos: Foram operados 20 ratos Wistar, pesando $260 \pm 14 \mathrm{~g}$ separados aleatoriamente nos grupos A1,A2, B1 e B2, com cinco animais cada. Todos eles foram mantidos em gaiolas individuais recebendo água e alimento ad libitum e receberam apenas dieta líquida no pré-operatório imediato por um período de 12 horas. A anestesia processou-se com pentobarbital por via intraperitoneal e a anti-sepsia do campo operatório foi feita com solução de povidona. Uma laparotomia mediana de $4 \mathrm{~cm}$ a partir do apêndice xifóide foi realizada. O duodeno foi submetido a secção transversal, processando-se a anastomose com fio de polipropileno 6-0 em pontos simples separados. A parede abdominal foi fechada em dois planos com fio de nylon 4-0.

Nos animais dos grupos A1 e A2 foi aplicada sobre a anastomose solução contendo o FCFb na dose de $20 \mathrm{mg}$, em veículo de colágeno solúvel imediatamente após o término da sutura. Nos animais dos grupos B1 e B2 (experimentais) um mililitro de solução salina foi aplicado sobre a anastomose. Os grupos A1 e B1 foram observados até o $5^{\circ}$ dia e os $\mathrm{A} 2$ e B2 até o $7^{\circ}$ dia de pós-operatório. Todos os animais foram mantidos em dieta oral líquida (sacarose 10\%) por um período de $48 \mathrm{hs}$ no pós-operatório imediato, retornando a dieta sólida (labina PurinaÒ) a partir daí. Após os períodos de observação, os animais foram mortos com superdose de anestésico. A anastomose duodenal foi submetida ao teste de resistência à pressão, procedendose a secção do piloro e da transição duodenojejunal introduzindo-se catéter de polietileno $\mathrm{n}^{\mathrm{o}} 4$ na sua luz proximal, aí fixado com fio de algodão 000 , e feita a ligadura para oclusão da extremidade distal do duodeno. Através de catéter interligado a manômetro de mercúrio, foi feita a insuflação de ar em fluxo contínuo de 0,5 litro por minuto, antes preenchendo-se a cavidade abdominal com solução salina $0,9 \%$. Considerou-se a pressão máxima de ruptura da alça duodenal, quando surgiu o borbulhamento na solução salina na sutura duodenal. Em seguida o duodeno foi ressecado e colocado em formol a $10 \%$ por $48 \mathrm{hs}$, incluído em parafina e cortado em secções de $5 \mathrm{~m}$. As lâminas foram desparafinizadas, diafanizadas e coradas com hematoxilina-eosina e o tricrômico de Masson. A avaliação histológica das lâminas foi feita utilizando-se um sistema digitalizador e analisador de imagens. A área total dos campos microscópicos foi observada utilizando-se microscópio óptico (Olimpus), cuja imagem foi capturada por câmera e digitalizada através de Software Image Pro-plus, versão 3.0 (Media Cybernetics - LP, USA). Cada campo digitalizado foi dividido em unidades de imagem denominadas picture elements ou pixels, com coordenadas definidas. Foram avaliados dez campos aleatórios de cada lâmina. Após selecionada a resolução desejada, os impulsos ópticos foram digitalizados, resultando em uma imagem de cada campo que foi armazenada e processada em sistema multimídia. As lâminas foram examinadas para quantificação dos elementos da reação inflamatória como quantidade de fibroblastos, de neutrófilos, neovasos, tecido de granulação e colágeno, sob a forma de densidade mínima, máxima e média.

Análise Estatística: os dados obtidos foram analisados e comparados através do teste $t$ de Student com significância 0,05 e os resultados foram expressos pela média \pm desvio padrão.

\section{RESULTADOS}

Os animais estudados sobreviveram sem complicações pós-operatórias, sendo todos sacrificados nos períodos previstos. No grupo Al (observação por 5 
dias), a pressão intraluminal suportada pelo duodeno foi de $52 \pm 14,4 \mathrm{mmHg}$ e no A2 (7 dias) a pressão atingiu o valor $140 \pm 34,8 \mathrm{mmHg}$. No grupo B1, que não fez uso de FCF $\beta$ e foi observado por 5 dias, a média da pressão foi de $33,6 \pm 15,2 \mathrm{mmHg}$, enquanto no grupo B2 (7 dias) a pressão intraluminal foi de $105 \pm 30,3$. Os dados completos e respectiva interpretação estatística encontra-se na tabela 1 .

Tabela 1 - Valores das pressões suportadas pelas alças duodenais expressas em mmHg.

\begin{tabular}{c|c|c|c|c}
\hline Rato número & \multicolumn{2}{|c|}{ GRUPO A } & \multicolumn{2}{c}{ GRUPO B } \\
\hline & A1(5 dias $)$ & A2 (7 dias) & B1 (5 dias) & B2 (7 dias) \\
\hline 1 & 70 & 185 & 40 & 160 \\
\hline 2 & 55 & 140 & 20 & 110 \\
\hline 3 & 40 & 95 & 35 & 75 \\
\hline 4 & 35 & 160 & 55 & 80 \\
\hline 5 & 60 & 120 & 18 & 100 \\
\hline Médias & $52 \pm 14,4^{*}$ & $140 \pm 34,8^{* *}$ & $33,6 \pm 15,2 \phi$ & $105 \pm 30,3$ \\
\hline
\end{tabular}

$* \mathrm{p}<0,05$ comparado com A2, B2.

** $\mathrm{p}=0,01$ comparado com B2

$\phi \mathrm{p}<0,05$ comparado com B2; $\mathrm{p}>0,05$ comparado com A1.

Quando foram computados os dados histopatológicos através de sistema digitalizado, que transformou a quantificação dos elementos da reação tecidual em valores de densidade média, observou-se que os dados colhidos acompanharam aqueles referentes à resistência dos tecidos em cicatrização. Assim, no grupo Al a densidade média foi de $93 \pm 9,3$, em A2 o valor observado foi de $181,8 \pm 27,6$. Esses valores foram significativamente mais elevados $(\mathrm{p}<0,05)$ que os dos grupos B1 e B2, como se verifica na tabela 2 .

Tabela 2 - Densidade média dos elementos histopatológicos do tecido duodenal em cicatrização.

\begin{tabular}{c|c|c|c|c}
\hline Rato número & \multicolumn{2}{|c|}{ GRUPO A } & \multicolumn{2}{c}{ GRUPO B } \\
\hline & A1(5 dias) & A2 (7 dias) & B1 (5 dias) & B2 (7 dias) \\
\hline 1 & 102,7 & 190,6 & 76,7 & 90,8 \\
\hline 2 & 89,5 & 201,4 & 55,9 & 102,6 \\
\hline 3 & 95,2 & 142,4 & 49,0 & 88,6 \\
\hline 4 & 100,9 & 166,9 & 67,1 & 105,5 \\
\hline 5 & 79,8 & 210,7 & 91,0 & 120,1 \\
\hline Médias & $93 \pm 9,3^{*}$ & $181,8 \pm 27,6^{* *}$ & $67,6 \pm 16,7 \phi$ & $101 \pm 12,9$ \\
\hline
\end{tabular}

* $\mathrm{p}<0,05$ comparado A2, B1; $\mathrm{p}=0,22$ comparado com B2

** $\mathrm{p}<0,05$ comparado com B1 e B2

$\phi \mathrm{p}=0,002$ comparado com B2

No grupo B1 chamou a atenção a densidade mínima de colágeno nas lâminas examinadas. Em todas elas, as fibras de colágeno apareciam desorganizadas, distribuídas aleatoriamente. Observou-se maior quantidade de neovasos, células inflamatórias, fibroblastos e fibras de colágeno no grupo A-1. As lâminas do grupo A2 mostraram sistematicamente grande celularidade à base principalmente de neutrófilos e macrófagos; a quantidade de fibroblastos, de fibras de colágeno e a neovascularização foram maiores que no grupo B-2. 


\section{DISCUSSÃO}

Aspecto importante, que deve ser levado em consideração, é o fato de que, apesar da rica vascularização duodenal, este órgão está sujeito a complicações conseqüentes à isquemia, manipulação cirúrgica e agressão cloridropéptica $^{11,12}$.

As fístulas duodenais são na sua grande maioria conseqüentes à condição patológica denominada "duodeno difícil". Segundo Schein e Decker ${ }^{13}$, as complicações do tratamento do coto duodenal difícil não têm merecido grande destaque, embora continuem ocorrendo e sendo dotadas de grande potencial mórbido. As casuísticas mais significativas sobre o assunto foram publicadas até a década de 70 , tendo em vista ter sido, até aquela época, a gastrectomia subtotal o tratamento padrão para as úlceras duodenais ${ }^{14}$. Com a diminuição da indicação do tratamento cirúrgico para a úlcera duodenal, aumentou a incidência de complicações, que passaram a incidir cada vez mais em pessoas mais idosas ${ }^{15}$. A incidência atual das fístulas duodenais está em torno de 1,5 a $3,0 \%,{ }^{16}$. Desse modo, os fatores que mais contribuem para a incidência das deiscências e fístulas duodenais são: as desvascularizações consequentes às extensas dissecções, a circulação terminal e a fibrose cicatricial nas úlceras duodenais crônicas ${ }^{16}$. Deve ser ressaltado igualmente que o duodeno apresenta outras características, que fazem com que seja um órgão sujeito a essas complicações, quais sejam: é um órgão predominantemente retroperitoneal, portanto sem a proteção da serosa peritoneal; recebe através da papila os sucos biliar e pancreático, fato que o torna mais susceptível a complicações mais graves na vigência de fístulas e deiscências; recebe ácido clorídrico do estômago, substância altamente agressiva à parede duodenal e aos fios de sutura. Essas características fizeram com que o duodeno fosse escolhido como o órgão alvo para o presente trabalho, podendo-se denominar as suturas e anastomoses do duodeno como de alto risco ${ }^{16}$.

$\mathrm{O} \mathrm{FCFb}$ mostrou-se eficaz no presente trabalho em melhorar a qualidade da cicatrização de lesão do duodeno ao estimular a neovascularização, a celularidade à base de fibroblastos, neutrófilos e macrófagos, bem como a formação de colágeno, mesmo que a observação tenha sido até o sétimo dia pós-operatório. Várias técnicas têm sido usadas para a quantificação da resistência anastomoses de alças intestinais, como extensômetro de resistência elétrica ${ }^{17}$, coluna de mercúrio e selo d'água ${ }^{18}$, resistência com insuflação ${ }^{20}$. Optou-se no presente estudo pela determinação da resistência da anastomose ainda na cavidade abdominal, com o objetivo de evitar a interferência de lesões acidentais da área em estudo durante a dissecção do duodeno. Witte e Barbul (21) afirmam que, para que uma cicatrização se faça a contento, são essenciais: boa irrigação, ausência de tensão, trauma tecidual mínimo, hemostasia adequada e técnica perfeita e rigorosa.

Com a finalidade de reduzir ao máximo a interferência subjetiva do patologista ao examinar as lâminas ao exame histopatológico, foi utilizado sistema digital de captação de imagens e quantificação dos achados histológicos em densidade média. Sistema semelhante foi utilizado por Schnaider et $\mathrm{al}^{26}$. Alguns outros métodos têm sido utilizados com resultados satisfatórios ${ }^{22,23,24,25}$.

Os fatores do crescimento constituem um grupo de peptídeos à semelhança de hormônios, que afetam uma grande variedade de sistemas biológicos, inclusive a cicatrização das feridas, quando administrados in vivo ou in vitro ${ }^{27}$. Com a descoberta dos fatores do crescimento derivados de plaquetas, do fator transformador beta e do $\mathrm{FCFb}$, tem sido possível demonstrar a melhora da cicatrização das feridas em certo número de modelos in vivo pela aplicação tópica nas feridas ${ }^{28}$. Muitos desses fatores de crescimento são produzidos por células reconhecidamente envolvidas na reparação das feridas. Estudos já comprovaram que o $\mathrm{FCFb}$ é um potente estimulador da angiogênese, é um mitógeno e substância quimiotáxica que atrai células endoteliais e fibroblastos. Esses efeitos foram observados com bastante evidência no presente estudo. Da mesma forma, tem-se demonstrado que o fator de crescimento de fibroblastos é um potente estimulador da produção de colagenase e do ativador do plasmonogênio, que facilitam a migração de células através da matriz do tecido em cicatrização ${ }^{28}$.

\section{CONCLUSÕES}

1 - De acordo com os dados obtidos do estudo em ratos, pôde-se concluir que o FCFb foi capaz de aumentar a resistência das feridas do duodeno suturadas e observadas após 5 e 7 dias de evolução.

2 - A aplicação tópica de $\mathrm{FCFb}$ em feridas do duodeno de ratos estimulou a neovascularização, a formação de fibroblastos e de fibras colágenas, melhorando os escores histológicos em relação ao controle.

\section{REFERÊNCIAS}

1. McGrath $\mathrm{MH}$. Peptide Growth Factors and wound healing. $\mathrm{Cl}$ Plast Surg 1990; 17:421-432.

2. Folkman J, Klagsbrun M. Angiogenic factors. Science 1987;235: 442-447

3. Hunt TK, LaVan FB. Enhancement of wound healing by growth factors. N Engl J Med 1989; 321:111-112.

4. Ross R. Platelet-derived growth factor. Annu Rev Med 1987; 38: 71-79.

5. Richard JL, Parer-Richard C, Daures JP. Effect of topical basic fibroblast growth factor on the healing of chronic diabetic reurotrophic ulcers of the foot. Diabetes Care 1995; 18:64-69.

6. Lynch SE, Nixon JC, Colvin RB. Role of platelet-derived growth factor in the wound healing: Synergistic effects with growth factors. Proc Natl Acad Sci USA 1987; 84: 7695-7697. 
7. Muscoe T, Cutler N, Allman R. A phase II study to evaluate recombinant platelet-derived growth factor-BB in the treatment of stage 3 to 4 pressure ulcers. Arch Surg 1994;129:212-219.

8. Davidson J. Growth factors in wound healing. Wound 1995; 7(suppl A): 53-64.

9. Deuel TF, Kawsahara RS, Muscoe TA. Growth factors and wound healing: platelet-derived growth factor as a model of cytokine. Ann Rev Med 1991; 42: 567-574.

10. Falanga V, Zitelli JA, Eaglstein WH. Wound healing. J Am Acad Dermatol 1988; 19:559-563.

11. Zollinger RM. Atlas of surgical operations. Mac millan Publishing Co. Inc. New York, 1975.

12. Tarazi R, Coutsoftides T, Steiger E. Gastric and duodenal cutaneous fistulas. World J Surg 1983; 7: 463-5.

13. Schein M, Decker GAG. Gastrointestinbal fistulas associated with large abdominal wall deffects. Experience with 43 patients. Br J Surg 1990; 77: 97-100.

14. Rose D, Yarborough MF, Canizaro PC. One hundred and fourteen fistulas of the gastrointestinal tract treated with total parenteral nutrition. Surg Gynecol obsted. 1986; 163:345

15. Dardai E, Pirityi S, Nagy L. Parenteral and enteral nutrition and the enterocutaneous treatment. Factors influencing the outcome of treatment. Acta Chir Hung 1991; 32: 305-308.

16. Reber HÁ, Roberts C, Way LW, et al. Management of external gastrointestinal fistulas. Ann Surg 1978; 188:460-470.

17. Naresse LE, Lucchiari PH, Angelili AYO, Burini RC, Rodrigues MAM, Curi PR, Kobayasi S. Estudo comparativo de anastomoses no intestino delgado de cão: Estudos da força de ruptura, hidroxoprolina tecidual e anatomopatológico. Acta Cir Bras 1988; 3:106-112.

18. Petroianu A, Souza sd, Martins SG, Alberti LR, Vasconcelos LS. Influência da vitamina $\mathrm{C}$ e da hidrocortisona sobre a tensão anastomótica jejunal em ratos. Acta Cir Bras 2000;15:215219

19. Forrest L. Current concepts in soft connective tissue wound healing (review). Br J Surg 1983; 70: 133-5.

20. Polônio B, Repta JCD, Novo NF, Jiliano Y, Nigro AJT. Anastomoses esôfago-esofágicas cervicais término-terminais por invaginação e com anel biodegradável: estudo comparativo em cães. Acta Cir Bras 1996;11:51-57.

21. Witte MB, Barbul A. General principles of wound healing. Surg Cl North Am 1997; 77: 509-528.

22. Aidos RD, Magalhães AM, Meneses Filho JF, Barone B, Goldenberg S. Efeitos da diatermia cirúrgica na cicatrização de anastomoses do intestino grosso. Estudo experimental em coelhos. Acta Cir Bras 1994;9:190194.

23. Naresse LE, Leite CVS, Rodrigues MAM, Angelili AYO, Minossi JG, Kobayasi S. Efeito da peritonite fecal na cicatrização do cólon distal no rato: avaliação anatomopatológica, estudo da força de ruptura e da hidroxiprolina tecidual. Acta Cir Bras $1993 ; 8: 48-53$

24. Azevedo JLMC, Goldenberg S, Simões MJ, Stavale JN. Estudo comparativo entre as anastomoses em plano único extramucoso e total, em colo de coelho. Acta Cir Bras 1990;5:6-12.

25. Simões JC, Polônio B, Scaeffer Neto C, Pupo CA, Auersvald A, Collaço LM. Aspectos morfológicos da reação tecidual da película celulósica inserida em esplenectomia parcial, hepatectomia parcial e no jejuno. Estudo experimental em cães. Acta Cir Bras 1990;5:88-93.

26. Schnaider TB, Lázaro da Silva A, Engelman MFB, Juliano Y, Novo NF, Schnaider GS, Schnaider CS. Estudo morfométrico do efeito do tenoxicam com água bidestilada ou com cloreto de sódio a $0,9 \%$ no endotélio venoso em coelhos. Acta Cir Bras 2002; 17:122-129.

27. Gospodarowicz D. Biological activities of fibroblast growth factor. Ann NY Acad Sci 1991; 638:1-8.

Medeiros AC, Dantas Filho AM, Aires Neto T, Lima FP, Azevedo IM, Alves SG. The basic fibroblast growth factor enhances the healing of duodenal anastomosis in rats. Acta Cir Bras [online] 2003 vol 18 suppl 1. Available in www.scielo.br/acb.

ABSTRACT: Objective: Analysis of histopatological alterations, and pressure resistence in duodenal anastomosis treated with basic fibroblast growth factor (FGF-b). Methods: Twenty Wistar rats were submited to a transversal duodenal section and subsequent anastomosis. They were randomly separated into four groups of five rats each: A1 and A2 (experimentals), in which FCFb was applied over the anastomosis; in B1 and B2 (controls), saline was use at the anastomosis site. The rodents were killed with an anesthetics overdosis, according to the following protocol: Al, B1 on $5^{\text {th }}$ postoperative day and A2, B2 on the $7^{\text {th }}$ one. A pressure resistence test of the anastomosis was done. The digitalized system Image ProPlus was used in order to analyse the mean density of the histopatological elements of healing duodenal tissues. Results: All the rats survived without complications. In the group A1 the intraluminal pressure was $52 \pm 14,4 \mathrm{mmHg}$ and in group A2 it was $140 \pm 34,8 \mathrm{mmHg}$. In the group B1 the pressure reached $33,6 \pm 15,2 \mathrm{mmHg}$ and in B2 it reached $105 \pm 30,3$. In group A1 the mean density of histopatological elements was $93 \pm 9,3$ and in A2 it was $181,8 \pm 27,6$. In the control groups B1 and B2 the mean densitys were $67,6 \pm 16,7$ and $101 \pm 12,9$ respectively. The statistical analysis detected a significant difference between the data of the experimental and control groups $(\mathrm{p}<0,05)$. Conclusion: the topical use of $\mathrm{FGFb}$ was able to enhance the resistence of duodenal anastomosis in rats observed and evaluated after five and seven postoperative days. The FCFb enhanced the neovascularization and the mean density of collagen, fibroblasts and inflammatory cells in the healing tissues.

KEYWORDS: Fibroblast growth factor. Healing. Anastomosis. Duodenum. Resistence. Collagen.

Correspondência:

Aldo da Cunha Medeiros

Av. Miguel Alcides Araújo 1889 - 59078-270 Natal-RN 